This item was submitted to Loughborough's Research Repository by the author.

Items in Figshare are protected by copyright, with all rights reserved, unless otherwise indicated.

Solving the characteristic initial-value problem for colliding plane gravitational and electromagnetic waves

PLEASE CITE THE PUBLISHED VERSION

LICENCE

CC BY-NC-ND 4.0

REPOSITORY RECORD

Alekseev, G.A., and J.B. Griffiths. 2019. "Solving the Characteristic Initial-value Problem for Colliding Plane Gravitational and Electromagnetic Waves”. figshare. https://hdl.handle.net/2134/687. 


\title{
Solving the characteristic initial value problem for colliding plane gravitational and electromagnetic waves
}

\author{
G. A. Alekseev ${ }^{1, *}$ and J. B. Griffiths ${ }^{2, \dagger}$ \\ ${ }^{1}$ Steklov Mathematical Institute, Gubkina 8, Moscow 117966, GSP-1, Moscow, Russia. \\ ${ }^{2}$ Department of Mathematical Sciences, Loughborough University, Loughborough, \\ Leics. LE11 3TU, U.K.
}

May 4, 2001

\begin{abstract}
A method is presented for solving the characteristic initial value problem for the collision and subsequent nonlinear interaction of plane gravitational or gravitational and electromagnetic waves in a Minkowski background. This method generalizes the monodromy transform approach to fields with nonanalytic behaviour on the characteristics inherent to waves with distinct wave fronts. The crux of the method is in a reformulation of the main nonlinear symmetry reduced field equations as linear integral equations whose solutions are determined by generalized ("dynamical") monodromy data which evolve from data specified on the initial characteristics (the wavefronts).
\end{abstract}

\section{Introduction}

The collision and subsequent nonlinear interaction between plane gravitational or gravitational and electromagnetic waves propagating with distinct wavefronts in a Minkowski background is a well formulated characteristic initial value problem. However, even the discovery of the integrability of the main field equations for this situation did not lead to a solution of this complex nonlinear problem. None of the existing solution generating methods have been found suitable for an effective construction of the solution in the wave interaction region starting from given characteristic initial data determined by the approaching waves.

The structure of the governing field equations for colliding plane waves, physical and geometrical interpretations, and various particular solutions and techniques, have been described in [1]. For colliding plane gravitational waves with aligned constant polarizations, the vacuum Einstein equations are reducible to the linear Euler-Poisson-Darboux equation. In this case, the corresponding characteristic initial value problem can be solved using the generalized version of Abel's transform [2].

However, when the polarizations of the approaching gravitational waves are not constant and aligned, or in the presence of electromagnetic waves, the governing equations are essentially nonlinear and are equivalent to the hyperbolic form of the Ernst equations. For this case, an appreciable number of particular solutions are known. These have been found using the "inverse" method in which a formal solution in the interaction region is first constructed and the corresponding characteristic initial data for the approaching waves is only determined subsequently. Recently, infinite hierarchies of exact vacuum and electrovacuum solutions with an arbitrary number of free parameters were found [3], and many of these are of the type appropriate for

${ }^{*}$ Email address: G.A.Alekseev@mi.ras.ru

${ }^{\dagger}$ E-mail: J.B.Griffiths@Lboro.ac.uk 
colliding plane waves. However, it is not a simple technical task to simplify these solutions for particular cases and to calculate the corresponding characteristic initial data.

For the analysis of the characteristic initial value problem for the vacuum hyperbolic Ernst equation, Hauser and Ernst [4] have generalized their group-theoretical approach (which had been developed earlier for stationary axisymmetric fields) and constructed a homogeneous Hilbert problem with corresponding matrix linear integral equations. Many aspects of this problem, including the existence and uniqueness of solutions and a detailed proof of the Geroch conjecture, were elaborated in [5].

However, a general scheme for the solution of various nonlinear initial and boundary value problems for integrable reductions of Einstein's equations had been developed in the framework of the monodromy transform approach [6]-[9]. This can be applied to both the characteristic initial value problem and the Cauchy problem for the hyperbolic case, as well as some boundary problems for the elliptic case. In this approach, every solution can be characterized by a set of functions of an auxiliary (spectral) parameter. These functions are interpreted as the monodromy data on the spectral plane of the fundamental solution of an auxiliary overdetermined linear system associated with the (nonlinear) field equations. These monodromy data are nonevolving (i.e. coordinate independent) and, generally, can be chosen arbitrarily or specified in accordance with the properties of the solution being sought. In particular, these data can be determined (at least in principle) from the initial or boundary data. In this scheme, the solution of the initial or boundary value problem is determined by the solution of some linear singular integral equations whose kernel is constructed using these (specified) monodromy data.

Recently a further generalization of this approach was derived in [10], in which a new linear integral equation form of various hyperbolic integrable reductions of Einstein equations was constructed. The scalar kernel of these quasi-Fredholm equations depends on the monodromy data in a different way - referred to as "dynamical monodromy data". Unlike the previous method, these data evolve, and their evolution is prescribed by the characteristic initial conditions. It appears that these new integral equations are better adapted to the construction of an effective solution of initial value problems because their coefficients carry more explicit information about the characteristic initial data and the corresponding analytical structures of the solutions desired.

In this paper, we present a new approach to the solution of the colliding plane wave problem and also describe a method that can be implemented in practice to derive explicit solutions. Our construction generalizes the monodromy transform approach whose formulation in the above mentioned papers was applicable only for fields that are analytically dependent on some special set of geometrically defined space-time coordinates. (One of these coordinates determines the measure of an area on the two-dimensional orbits of the space-time isometry group. The other is its harmonic conjugate.) However, for colliding plane waves propagating with distinct wave fronts on a Minkowski background, this analyticity is obviously violated on the wave fronts. Moreover, the physically accepted matching conditions (the "colliding plane wave conditions") imply regular behaviour of the field components near the wave fronts in some appropriate null coordinates [1]. In terms of geometrically defined coordinates, this regularity leads to a specific singular behaviour of the coefficients of the associated linear system on these hypersurfaces, where the first derivatives of the field components become infinite. This gives rise to crucial consequences for the previous formulation. Additional singularities appear on the spectral plane for some auxiliary functions. Also, the integral representations diverge at the singular points, while the normalization of the solutions remains a necessity. Another important phenomenon which arises in these singular cases is that the nonevolving monodromy data, which continue to exist, lose their the most important property - their unambiguous characterization of the solution.

The solution of these problems arises from our recent observation that one of two linear integral "evolution equation" forms of the field equations derived in [10] admits a generalization to the singular case. It is also important that the dynamical monodromy data can still be used 
in this case to characterize the solutions. In this paper we present the generalized linear quasiFredholm integral "evolution equation", which covers the singular case and opens a direct way for the construction of solutions for colliding plane waves from given characteristic initial data. We also briefly discuss some applications.

\section{Associated linear system with spectral parameter}

We base our construction on the Kinnersley-like overdetermined linear system with a free complex parameter $w$ for a $N \times N$ matrix function $\boldsymbol{\Psi}(\xi, \eta, w)$ whose integrability conditions are equivalent to the hyperbolic space-time symmetry reduction for $N=2$ of the vacuum Einstein equations and for $N=3$ of the electrovacuum Einstein-Maxwell equations -

$$
\left\{\begin{array}{lll}
2 i(w-\xi) \partial_{\xi} \mathbf{\Psi}=\mathbf{U}(\xi, \eta) \mathbf{\Psi}, & \operatorname{rank} \mathbf{U}=1, & \operatorname{tr} \mathbf{U}=i \\
2 i(w-\eta) \partial_{\eta} \mathbf{\Psi}=\mathbf{V}(\xi, \eta) \boldsymbol{\Psi}, & \operatorname{rank} \mathbf{V}=1, & \operatorname{tr} \mathbf{V}=i
\end{array}\right.
$$

in which the null coordinates $\xi, \eta$ are certain linear combinations of geometrically defined nonnull coordinates mentioned in the introduction. These equations must be supplemented with the additional constraints on its matrix integral $[7,9]$

$$
\left\{\begin{array}{l}
\mathbf{\Psi}^{\dagger} \mathbf{W} \mathbf{\Psi}=\mathbf{W}_{0}(w), \\
\mathbf{W}_{0}^{\dagger}(w)=\mathbf{W}_{0}(w),
\end{array} \quad \frac{\partial \mathbf{W}}{\partial w}=4 i \boldsymbol{\Omega}\right.
$$

where ${ }^{\dagger}$ denotes Hermitian conjugation $\left(\mathbf{W}_{0}^{\dagger}(w) \equiv \overline{\mathbf{W}_{0}^{T}(\bar{w})}\right)$ and $\boldsymbol{\Omega}$ is a constant matrix. For $N=2,3$, the only nonzero components of $\boldsymbol{\Omega}$ are $\boldsymbol{\Omega}^{12}=1$ and $\boldsymbol{\Omega}^{21}=-1$. For $N=3$, the condition $\mathbf{W}^{33}=1$ should be also satisfied. The real null coordinates $\xi, \eta$ are certain linear combinations of geometrically defined nonnull coordinates, say $\alpha, \beta$, mentioned in the introduction.

For any solution of these conditions, the components of $\mathbf{U}, \mathbf{V}$ and $\mathbf{W}$ can be identified with certain metric components and the electromagnetic potential and their derivatives. Without loss of generality, we impose the normalization conditions at the point denoted by $\left(\xi_{\times}, \eta_{\times}\right)$at which the waves collide. These are $\boldsymbol{\Psi}\left(\xi_{\times}, \eta_{\times}, w\right)=\mathbf{I}$ and $\mathbf{W}_{0}(w)=4 i\left(w-\beta_{\times}\right) \boldsymbol{\Omega}+\operatorname{diag}\left(4 \alpha_{\times}^{2}, 4,1\right)$, where $\alpha_{\times}=\left(\xi_{\times}-\eta_{\times}\right) / 2$ and $\beta_{\times}=\left(\xi_{\times}+\eta_{\times}\right) / 2$. A shift of origin and a rescaling of the coordinates $\xi$ and $\eta$ allow us to specify below $\xi_{\times}=1$ and $\eta_{\times}=-1$, so that $\alpha_{\times}=1, \beta_{\times}=0$.

\section{The colliding plane wave problem}

For plane gravitational or gravitational and electromagnetic waves with distinct wavefronts which collide in a Minkowski background, it is well known [1] that there exist global null coordinates $(u, v)$ such that the O'Brien-Singe matching conditions imposed on the wavefronts $u=0$ and $v=0$ lead to a well posed characteristic initial value problem. However, the regularity of this problem in these global coordinates in the interaction region $(u \geq 0, v \geq 0)$ implies special relations between these coordinates and the coordinates $(\xi, \eta)$ which are nonanalytical on the boundaries. It is possible to use the coordinate freedom to put

$$
\xi=1-2 u^{n_{+}}, \quad \eta=-1+2 v^{n_{-}},
$$

where $n_{ \pm} \geq 2$ are specified as part of the initial data. Thus, the important specific of this characteristic initial value problem is that, in terms of geometrically defined coordinates $(\xi, \eta)$, the first derivatives of the field components should be discontinuous and even unbounded on the wavefronts and at the point of collision. 


\section{The analytical structure of $\Psi$ on the spectral plane}

Everywhere below, $\boldsymbol{\Psi}$ denotes the fundamental solution of the associated linear system described above which is normalized at the point of collision $(u=0, v=0$, i.e. $(\xi=1, \eta=-1)$. As in the regular case [7,9], the structure of the coefficients of the associated linear system for $\boldsymbol{\Psi}$ implies that the normalized fundamental solution $\boldsymbol{\Psi}(\xi, \eta, w)$ and its inverse possess four branch points on the spectral plane $w$, namely at $w=\xi, w=\eta, w=1$ and $w=-1$. The order of these points and our choice of the cuts $L_{ \pm}$joining them are indicated in the figure

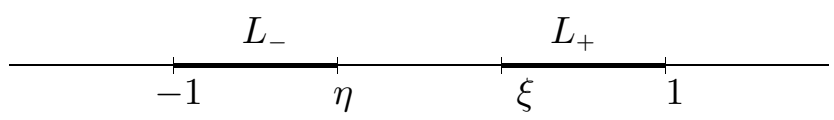

Near these singular points, and on the cuts $L_{ \pm}$, the components of $\boldsymbol{\Psi}$ and its inverse (as functions of $w$ for given $u \geq 0$ and $\mathrm{v} \geq 0$ ) possess in general the local structure:

$$
\mathbf{\Psi}(\xi, \eta, w)=\widetilde{\boldsymbol{\Psi}}_{ \pm}(\xi, \eta, w) \otimes \mathbf{k}_{ \pm}(w)+\mathbf{M}_{ \pm}(\xi, \eta, w)
$$

where, as in the regular case, the coordinate independent components of the row vectors $\mathbf{k}_{ \pm}(w)$ constitute the "projective vectors" of the monodromy data. Their components, as well as the components of the matrices $\mathbf{M}_{ \pm}(\xi, \eta, w)$, are regular (holomorphic) on the cuts $L_{ \pm}$with the corresponding index. In the analytical case we always have $\widetilde{\boldsymbol{\psi}}_{+}(\xi, \eta, w)=\sqrt{\frac{w-1}{w-\xi}} \boldsymbol{\psi}+(\xi, \eta, w)$ and $\widetilde{\boldsymbol{\psi}}_{-}(\xi, \eta, w)=\sqrt{\frac{w+1}{w-\eta}} \boldsymbol{\psi}_{-}(\xi, \eta, w)$ where the vectors $\boldsymbol{\psi}_{+}$and $\boldsymbol{\psi}_{-}$are holomorphic on the cuts $L_{+}$ and $L_{-}$respectively. In the general case, the components of the column vectors $\widetilde{\psi}_{ \pm}(\xi, \eta, w)$ also have branch points at the endpoints of the corresponding cuts $L_{ \pm}$, but the character of their singularities at the points $w=1$ and $w=-1$ respectively (as well as the powers $n_{+}$and $n_{-}$ defined above) are determined by the initial data.

\section{The integral "evolution" equations and the solution of the problem}

To set up the characteristic initial value problem, we introduce two matrix functions which are the normalized fundamental solutions of the linear ordinary differential equations - the restrictions of the associated linear system to the characteristics $\xi=1$ and $\eta=-1$ :

$$
\begin{array}{llrl}
2 i(w-\xi) \partial_{\xi} \boldsymbol{\Psi}_{+}=\mathbf{U}(\xi,-1) \cdot \boldsymbol{\Psi}_{+}, & & \boldsymbol{\Psi}_{+}(1, w)=\mathbf{I} \\
2 i(w-\eta) \partial_{\eta} \boldsymbol{\Psi}_{-}=\mathbf{V}(1, \eta) \cdot \boldsymbol{\Psi}_{-}, & & \boldsymbol{\Psi}_{-}(-1, w)=\mathbf{I}
\end{array}
$$

in which the coefficients are determined by the initial data for the fields on the corresponding characteristics. These matrices should be the characteristic initial data for the required solution for $\boldsymbol{\Psi}(\xi, \eta, w)$ :

$$
\boldsymbol{\Psi}_{+}(\xi, w) \equiv \boldsymbol{\Psi}(\xi,-1, w), \quad \mathbf{\Psi}_{-}(\eta, w) \equiv \boldsymbol{\Psi}(1, \eta, w)
$$

The analytical structures of $\boldsymbol{\Psi}_{ \pm}$on the spectral plane are very similar to those of $\boldsymbol{\Psi}$. Namely, $\boldsymbol{\Psi}_{ \pm}(w=\infty)=\mathbf{I}, \boldsymbol{\Psi}_{+}$is holomorphic outside $L_{+}$and $\boldsymbol{\Psi}_{-}$outside $L_{-}$, and their local structures on these cuts are given by

$$
\begin{array}{ll}
L_{+}: & \boldsymbol{\Psi}_{+}(\xi, w)=\widetilde{\Psi}_{0+}(\xi, w) \otimes \mathbf{k}_{+}(w)+\mathbf{M}_{0+}(\xi, w), \\
L_{-}: & \boldsymbol{\Psi}_{-}(\eta, w)=\widetilde{\boldsymbol{\Psi}}_{0_{-}}(\eta, w) \otimes \mathbf{k}_{-}(w)+\mathbf{M}_{0-}(\eta, w)
\end{array}
$$

where $k_{ \pm}(w)$ are the same as for $\mathbf{\Psi}$, and $\mathbf{M}_{0+}(\xi, w)$ and $\mathbf{M}_{0-}(\eta, w)$ are holomorphic on $L_{+}$and $L_{-}$respectively.

We now introduce, in analogy with the regular case [10], the "evolution" or "scattering" matrices $\boldsymbol{\chi}_{ \pm}(\xi, \eta, w)$, representing $\mathbf{\Psi}(\xi, \eta, w)$ in two alternative forms

$$
\begin{aligned}
& \boldsymbol{\Psi}(\xi, \eta, w)=\chi_{+}(\xi, \eta, w) \cdot \boldsymbol{\Psi}_{+}(\xi, w) \\
& \boldsymbol{\Psi}(\xi, \eta, w)=\chi_{-}(\xi, \eta, w) \cdot \boldsymbol{\Psi}_{-}(\eta, w) .
\end{aligned}
$$


The crucial point is that the components of the matrix $\chi_{+}(\xi, \eta, w)$ are holomorphic on $L_{+}$and possess a jump on $L_{-}$only, while the components of the matrix $\chi_{-}(\xi, \eta, w)$ are holomorphic on $L_{-}$and possess a jump on $L_{+}$. From the above it is clear also, that these jumps are represented by highly degenerate matrices and that $\boldsymbol{\chi}_{ \pm}(\xi, \eta, w=\infty)=\mathbf{I}$. These properties permit us to represent $\chi_{ \pm}$as Cauchy integrals of the form

$$
\begin{aligned}
& \boldsymbol{\chi}_{+}(\xi, \eta, w)=\mathbf{I}+\frac{1}{\pi i} \int_{L_{-}} \frac{\left[\widetilde{\boldsymbol{\Psi}}_{-}\right]_{\zeta_{-}} \otimes \mathbf{m}_{-}\left(\xi, \zeta_{-}\right)}{w-\zeta_{-}} d \zeta_{-} \\
& \boldsymbol{\chi}_{-}(\xi, \eta, w)=\mathbf{I}+\frac{1}{\pi i} \int_{L_{+}} \frac{\left[\widetilde{\boldsymbol{\Psi}}_{+}\right]_{\zeta_{+}} \otimes \mathbf{m}_{+}\left(\xi, \zeta_{+}\right)}{w-\zeta_{+}} d \zeta_{+}
\end{aligned}
$$

where [...] is the jump (a half of the difference between the left and right limits) of a function on a cut and

$$
\begin{aligned}
& \mathbf{m}_{-}(\xi, w)=\mathbf{k}_{-}(w) \cdot \boldsymbol{\Psi}_{+}^{-1}(\xi, w) \\
& \mathbf{m}_{+}(\eta, w)=\mathbf{k}_{+}(w) \cdot \boldsymbol{\Psi}_{-}^{-1}(\eta, w)
\end{aligned}
$$

represent a new (evolving) kind of monodromy data introduced for the regular case in [10] and called there the "dynamical" monodromy data. It is necessary to note here our conjecture of the convergency of the Cauchy integrals for $\chi_{ \pm}$at $w=-1$ and $w=1$ respectively which is confirmed for particular examples for any $n_{ \pm} \geq 2$.

The above alternative representations for $\boldsymbol{\Psi}$ should satisfy an obvious condition $\boldsymbol{\chi}_{+} \boldsymbol{\Psi}_{+} \equiv$ $\chi_{-} \boldsymbol{\Psi}_{-}$. This condition considered on the cuts $L_{ \pm}$together with the constructed integral representations for $\chi_{ \pm}$leads to the linear integral equations (for brevity we omit here the parametric dependence of all objects upon $\xi$ and $\eta$ ):

$$
\begin{gathered}
\boldsymbol{\phi}_{+}\left(\tau_{+}\right)-\int_{L_{-}} S_{+}\left(\tau_{+}, \zeta_{-}\right) \boldsymbol{\phi}_{-}\left(\zeta_{-}\right) d \zeta_{-}=\boldsymbol{\phi}_{0+}\left(\tau_{+}\right) \\
\boldsymbol{\phi}_{-}\left(\tau_{-}\right)-\int_{L_{+}} S_{-}\left(\tau_{-}, \zeta_{+}\right) \boldsymbol{\phi}_{+}\left(\zeta_{+}\right) d \zeta_{+}=\boldsymbol{\phi}_{0-}\left(\tau_{-}\right)
\end{gathered}
$$

where $\tau_{+}, \zeta_{+} \in L_{+}, \tau_{-}, \zeta_{-} \in L_{-}$and the vector functions $\boldsymbol{\phi}_{+}\left(\tau_{+}\right), \boldsymbol{\phi}_{-}\left(\tau_{-}\right)$and their initial values $\boldsymbol{\phi}_{0+}\left(\tau_{+}\right), \boldsymbol{\phi}_{0-}\left(\tau_{-}\right)$are the jumps $\left[\widetilde{\boldsymbol{\psi}}_{+}\right]_{\tau_{+}},\left[\widetilde{\boldsymbol{\psi}}_{-}\right]_{\tau_{-}}$and $\left[\widetilde{\boldsymbol{\psi}}_{0+}\right]_{\tau_{+}},\left[\widetilde{\boldsymbol{\psi}}_{0-}\right]_{\tau_{-}}$respectively. The scalar kernels are given by

$$
\begin{aligned}
& S_{+}\left(\xi, \tau_{+}, \zeta_{-}\right)=\frac{1}{i \pi\left(\zeta_{-}-\tau_{+}\right)}\left(\mathbf{m}_{-}\left(\xi, \zeta_{-}\right) \cdot \mathbf{\phi}_{0+}\left(\xi, \tau_{+}\right)\right) \\
& S_{-}\left(\eta, \tau_{-}, \zeta_{+}\right)=\frac{1}{i \pi\left(\zeta_{+}-\tau_{-}\right)}\left(\mathbf{m}_{+}\left(\eta, \zeta_{+}\right) \cdot \boldsymbol{\phi}_{0-}\left(\eta, \tau_{-}\right)\right)
\end{aligned}
$$

As in the regular case, the coefficients of the above integral equations are determined by the initial data. However, these generalized "evolution equations" possess a more complicated singular structure. They also can be easily decoupled into two independent equations for $\boldsymbol{\phi}_{+}$ and $\phi_{-}$, but we omit these calculations here.

Our construction of solutions for colliding plane waves begins with the constants $n_{ \pm} \geq 2$ which determine the degree of non-smoothness of the fields on the wavefronts and the characteristic initial data for the fields in terms of the Ernst potentials $\mathcal{E}(u, v), \Phi(u, v)$ which characterise every solution. These data, viz. $\mathcal{E}_{+}(u), \Phi_{+}(u), \mathcal{E}_{-}(v)$, and $\Phi_{-}(v)$, should be chosen to satisfy the normalization conditions $\mathcal{E}_{ \pm}(0)=-1, \Phi_{ \pm}(0)=0$ and two wavefront regularity conditions [1]: $\left|\mathcal{E}_{ \pm}^{\prime}(0)\right|^{2}+4\left|\Phi_{ \pm}^{\prime}(0)\right|^{2}=8\left(1-1 / n_{ \pm}\right)$. With this data we have to solve the ordinary differential equations for $\boldsymbol{\Psi}_{ \pm}$and the integral equations for $\boldsymbol{\phi}_{ \pm}$. The Ernst potentials of the sought-for 
solution can be evaluated then as

$$
\begin{aligned}
\mathcal{E}(u, v) & =\mathcal{E}_{+}(u)+\frac{2}{\pi} \int_{L_{-}}\left(\mathbf{e}_{1} \cdot \boldsymbol{\phi}-\left(\zeta_{-}\right)\right)\left(\mathbf{m}_{-}\left(\zeta_{-}\right) \cdot \mathbf{e}_{2}\right) d \zeta_{-} \\
& =\mathcal{E}_{-}(v)+\frac{2}{\pi} \int_{L_{+}}\left(\mathbf{e}_{1} \cdot \boldsymbol{\phi}+\left(\zeta_{+}\right)\right)\left(\mathbf{m}_{+}\left(\zeta_{+}\right) \cdot \mathbf{e}_{2}\right) d \zeta_{+} \\
\Phi(u, v) & =\Phi_{+}(u)-\frac{2}{\pi} \int_{L_{-}}\left(\mathbf{e}_{1} \cdot \boldsymbol{\phi}{ }_{-}\left(\zeta_{-}\right)\right)\left(\mathbf{m}_{-}\left(\zeta_{-}\right) \cdot \mathbf{e}_{3}\right) d \zeta_{-} \\
& =\Phi_{-}(v)-\frac{2}{\pi} \int_{L_{+}}\left(\mathbf{e}_{1} \cdot \boldsymbol{\phi}+\left(\zeta_{+}\right)\right)\left(\mathbf{m}_{+}\left(\zeta_{+}\right) \cdot \mathbf{e}_{3}\right) d \zeta_{+}
\end{aligned}
$$

where $\mathbf{e}_{1}=\{1,0,0\}, \mathbf{e}_{2}=\{0,1,0\}$ and $\mathbf{e}_{3}=\{0,0,1\}$.

As a simple test, we consider $n_{+}=n_{-}=2$ and chose

$$
\mathcal{E}_{+}=-(1-u)^{2}, \quad \mathcal{E}_{-}=-(1-v)^{2}
$$

For these vacuum data our calculations lead immediately to the Khan-Penrose solution for the collision of impulsive gravitational waves with colinear polarizations. Similar calculations for the initial data $\left(\delta_{ \pm}\right.$are real constants)

$$
\mathcal{E}_{+}=-1+2 e^{i \delta_{+}} u-u^{2}, \quad \mathcal{E}_{-}=-1+2 e^{i \delta_{-}} v-v^{2}
$$

give the Nutku-Halil solution for the collision of these waves with noncolinear polarizations. The initial data

$$
\mathcal{E}_{+}=-1, \quad \Phi_{+}=u, \quad \mathcal{E}_{-}=-1, \quad \Phi_{-}=v
$$

lead to the Bell-Szekeres solution for the collision of electromagnetic step waves with aligned polarizations. When the polarizations of these waves is nonaligned, we change the data above to $\Phi_{-}=v e^{i \gamma},(\gamma$ is a real constant $)$. This produces

$$
\mathcal{E}(u, v)=-1, \quad \Phi(u, v)=u \sqrt{1-v^{2}}+v \sqrt{1-u^{2}} e^{i \gamma}
$$

which leads to a nontrivial (nondiagonal) metric (see [11]). Clearly, various new solutions can be found in the same way for different choices of the initial data.

\section{Acknowledgments}

The work of GA was partly supported by the EPSRC, by the INTAS grant 99-1782 and by the grants 99-01-01150, 99-02-18415 from the RFBR.

\section{References}

[1] J. B. Griffiths, Colliding plane waves in general relativity, (Oxford University Press, 1991).

[2] I. Hauser and F. J. Ernst, J. Math. Phys., 30, 872, 2322 (1989).

[3] G. A. Alekseev and J. B. Griffiths, Phys. Rev. Lett. 84, 5247 (2000).

[4] I. Hauser and F. J. Ernst, J. Math. Phys., 31, 871, (1990); 32, 198 (1991).

[5] I. Hauser and F. J. Ernst, Gen. Rel. Grav., 33, 195 (2001).

[6] G. A. Alekseev, Sov. Phys. Dokl. 30, 565 (1985).

[7] G. A. Alekseev, Proc. Steklov Inst. Maths. 3, 215 (1988). 
[8] G. A. Alekseev, Proceedings of the workshop NEEDS'92, p5 (World Scientific, Singapore, 1993).

[9] G. A. Alekseev, Proceedings of the workshop "Nonlinearity, Integrability and all that: Twenty years after NEEDS'79", p12 (World Scientific, Singapore, 2000); gr-qc/9911045.

[10] G. A. Alekseev, in preparation.

[11] J. B. Griffiths, In Galaxies, axisymmetric systems and relativity, (ed. M. A. H.MacCallum), p119 (Cambridge University Press, 1985). 